\title{
L'HOMME L'Homme
}

Revue française d'anthropologie

160 | octobre-décembre 2001

Droit, coutume, mémoire

\section{Une double peine}

La condition sociale des immigrés malades du sida

Didier Fassin

\section{OpenEdition}

Journals

Édition électronique

URL : http://journals.openedition.org//homme/129

DOI : $10.4000 /$ /homme.129

ISSN : 1953-8103

Éditeur

Éditions de l'EHESS

\section{Édition imprimée}

Date de publication : 1 janvier 2001

Pagination : 137-162

ISBN : 2-7132-1391-6

ISSN : 0439-4216

Référence électronique

Didier Fassin, «Une double peine », L'Homme [En ligne], 160 | octobre-décembre 2001, mis en ligne le

12 juin 2003, consulté le 30 avril 2019. URL : http://journals.openedition.org/lhomme/129; DOI :

10.4000/lhomme.129

〔c École des hautes études en sciences sociales 


\title{
Une double peine \\ La condition sociale des immigrés malades du sida
}

\author{
Didier Fassin
}

L'

ÉPIDÉMIE se présentant toujours comme un péril venu d'ailleurs, l'immigration offre à sa propagation un cadre d'interprétation qui s'impose au monde social avec la force de l'évidence, que ce soit à travers la figure fantasmatique de l'étranger menaçant qui en serait le vecteur ou sous la forme apparemment plus neutre des mouvements de populations qui la favoriseraient. Mais la maladie, loin d'être l'événement qui susciterait cette représentation stigmatisante, vient au contraire remplir un espace de disqualification préexistant, même virtuellement, où peuvent aussi trouver place la délinquance ou le chômage que l'immigré est toujours susceptible de propager selon des modalités volontiers imaginées en termes épidémiologiques de contamination et de diffusion. La menace pathogène vient ainsi s'ancrer dans des populations présupposées dangereuses (Clatts \& Mutchler 1989). L'épidémie se différencie toutefois de ces autres périls en ce qu'elle met à l'épreuve le corps physique tout autant que le corps social, exerçant sur l'un comme sur l'autre son pouvoir délétère. Les frontières mises en danger par les étrangers ne sont pas seulement celles du territoire national, elles sont aussi celles du territoire corporel.

Le sida, parce qu'il s'est d'emblée inscrit dans un double registre de la transgression (sexuelle) et de l'agression (par l'autre), s'est avéré une illustration remarquablement efficace de cette représentation d'une liaison dangereuse entre épidémie et immigration, aux États-Unis où la stigmatisation des Haïtiens a été particulièrement marquée comme en Russie où des Africains ont été expulsés après avoir été découverts malades du sida, en Europe où certains pays ont sélectionné les étudiants étrangers en fonction de leur statut sérologique comme en Afrique où les prostituées étrangères ont

L'enquête dont les données sont présentées ici fait partie d'un programme de recherche, intitulé «Expérience et construction de la maladie. Une étude sociologique et anthropologique du sida des migrants d'origine africaine dans la région parisienne » et financé par la Fondation pour la recherche médicale dans le cadre du Sidaction. Je remercie Bernadette Rwegera pour son aide dans la collecte et l'interprétation des entretiens recueillis par l'intermédiaire de l'association, et plus particulièrement la troisième biographie de ce texte. 
souvent fait l'objet de mesures répressives (Fassin 1996a). On ne s'étonnera pas, dans ces conditions, qu'il ait été si difficile de dire et même de penser ensemble ces deux termes - épidémie et immigration - notamment dans le contexte français où le souci légitime d'éviter les amalgames se doublait d'une cécité particulière à l'endroit de la "question immigrée» (Fassin 1999a). Exemplaire, le cas français n'est toutefois pas isolé et cette question est longtemps demeurée une sorte de point aveugle, et parfois douloureux, de l'histoire du sida dans la plupart des pays occidentaux (Haour-Knipe $\&$ Rector 1996). Après plus d'une décennie de silence presque complet et d'inaction à la mesure de ce silence, l'interdit a toutefois été contourné sur deux fronts distincts.

D'une part, une approche par la "culture " proposant des modèles ad hoc afin d'expliquer la rapidité de la progression et les échecs de la prévention (Farmer 1999) : les thèmes de la "promiscuité sexuelle " et du "refus du préservatif ", déjà utilisés pour rendre compte de l'explosion épidémique sur le continent africain, ont été resservis à propos des immigrés installés en Europe. D’autre part, une lecture en termes de "vulnérabilité » s'attachant à montrer les facteurs de fragilisation de ces populations face au risque d'infection (Delaunay 1999) : pour partie en réaction aux interprétations comportementalistes, il s'agissait, en s'appuyant là encore sur des notions développées dans le contexte africain, d'extrapoler le modèle physiopathologique faisant des maladies sexuellement transmissibles un cofacteur du sida à un modèle anthropologique prenant en considération les situations de précarité et les relations de genre susceptibles de les favoriser. Bien que différentes dans leurs préalables théoriques aussi bien que dans leurs conséquences pratiques, ces deux tentatives restent toutefois, pour l'essentiel, centrées autour de la question de la contamination, et donc du risque, dont on sait qu'elle peut difficilement être dissociée du thème de la dangerosité (Hahn, Eirmbter \& Jacob 1994). La mise en cause de la culture ou de la vulnérabilité dans la transmission du virus tend à constituer l'étranger comme une possible menace.

Mais qu'en est-il des malades eux-mêmes? En quoi l'expérience du sida estelle affectée par l'expérience de l'immigration? Ou, à l'inverse, en quoi souffrir de cette maladie transforme-t-il la vie des immigrés ? Les rares études qui traitent de l'épidémie parmi les populations étrangères vivant en France l'abordent sous l'angle des "représentations de la maladie » (Trachet \& Diallo 1994), soulignant ainsi sa spécification culturelle et la soustrayant à l'analyse des conditions d'existence des malades. Cette construction est d'autant plus marquée qu'elle se fait le plus souvent à partir de protocoles d'enquête, par passage de questionnaires ou réalisation de "focus groups", qui mettent doublement à distance les réalités quotidiennes des patients : d'une part, en s'adressant à des personnes non confrontées elles-mêmes ou à travers des proches à la maladie, de surcroît souvent rencontrées collectivement; d'autre part, en proposant des formulations artificiellement déclinées en "connaissances, attitudes et comportements ", bien loin des appréhensions cognitives et affectives opérant dans des situations concrètes ${ }^{1}$.

1. Rapports non datés de l'Unité de réflexion et d'action des communautés africaines sur "Communautés africaines et sida. Mythes et réalités ", et d'Afrique partenaires services sur « Perception du sida par les populations migrantes sahéliennes ». 
Quant aux études cliniques qui portent effectivement sur des malades et sont menées en général dans le cadre de consultations ethnopsychiatriques, elles mettent surtout l'accent sur la dimension magique des interprétations desquelles découlent ensuite les prescriptions thérapeutiques: les "étiologies sorcières" appellent ainsi des «sacrifices » supposés être pertinents compte tenu de "l'appartenance du patient à son monde culturel traditionnel $»^{2}$. Ainsi, quel qu'en soit le dispositif méthodologique, ces travaux ont pour effet de situer l'expérience du malade dans un univers de croyances où la tradition tient lieu d'historicité, où le symbolique prend le pas sur le matériel, où l'invocation de la sorcellerie permet d'éluder l'ordinaire de l'existence. De ce qu'il en est d'être immigré et/ou étranger lorsqu'on est malade du sida et de ce qu'il en est d'avoir le sida lorsque l'on est immigré et/ou étranger, il n'est guère question.

Pourtant la maladie constitue bien une épreuve particulière dans une expérience d'immigration, tout comme le fait d'être immigré modifie profondément l'expérience de la maladie. Bien plus qu'une question de spécificité culturelle que déterminerait l'origine différente ou de vulnérabilité particulière qui fragiliserait nécessairement l'étranger, il s'agit d'une tension entre deux conditions, celle de malade et celle d'immigré. C'est ainsi qu'il faut entendre l'expression "contradiction incorporée " qu'utilise Abdelmalek Sayad (1999a : 260) pour dire la relation entre maladie et immigration: "C'est à l'occasion de la maladie et de ses suites que se donnent le mieux à voir les contradictions constitutives de la condition même de l'immigré». Écrivant ces lignes au début des années 80 , il se référait en particulier à l'incapacité physique qu'entraîne l'apparition d'une affection ou d'un accident chez des personnes dont la seule raison d'être dans le pays d'accueil était leur activité productive : le corps devenait donc le lieu d'expression d'une contradiction profonde entre l'attente sociale à l'égard de l'immigré et la réalité de son impuissance. Dans un temps où le rapport entre demande de maind'œuvre étrangère et marché de l'emploi s'est distendu, le taux de chômage étant, chez les hommes de nationalité non européenne, trois fois plus élevé que la moyenne nationale, la tension entre la condition de malade et la condition d'immigré s'est déplacée, mais elle s'est également généralisée au sens où elle implique désormais un ensemble complexe de significations sociales qui excède la seule reconnaissance par le travail. Cette tension et les contradictions nouvelles sur lesquelles elle débouche sont l'objet de ce texte.

Condition. Le terme n'est peut-être pas sans ambiguïté : il faut l'entendre ici à la fois dans la dimension générique universelle que lui donne Hannah Arendt (1983 : 41-42) lorsqu'elle parle de "condition humaine » et dans la figure historique singulière que désigne Robert Castel (1995 : 323-325) quand il évoque la "condition ouvrière». S'agissant ici de l'expérience d'une affection grave, le pathos décontextualisé de la "condition de malade" doit être sans cesse confronté aux réalités sociales de la "condition d'immigré ». Le sida a d'ailleurs

2. Comptes rendus des séminaires «VIH et ethnopsychiatrie» du Centre Georges Devereux, 16 janvier et 7 mai 1996 . 
été volontiers traité par les anthropologues comme une " condition » en soi, sorte de mise à l'épreuve de l'humanité indépendamment de tout contexte historique et social particulier. À cet égard, la comparaison d'articles écrits à quelques années d'intervalle par les mêmes auteurs révèle comment on est passé de la qualification du sida comme "souffrance humaine" (Farmer \& Kleinman 1989), intemporellement associée à l'impureté et à la mort, à sa reconnaissance comme «souffrance sociale» (Kleinman 1995), produit de la "violence structurelle» (Farmer 1997), tant politique qu'économique, des sociétés contemporaines, en particulier dans le tiers monde. L'étude de la condition sociale de l'immigré malade, dont il est ici question, relève à l'évidence moins de l'anthropologie médicale illustrée dans le premier de ces textes que de l'économie politique défendue dans les seconds.

L'enquête visant à explorer cette relation entre la condition de malade et la condition d'immigré a été menée en Seine-Saint-Denis. Elle porte sur des malades du sida dont la plupart sont originaires d'Afrique subsaharienne, mais certains également du Maghreb et d'Haïti. Tous sont à la fois immigrés et étrangers, ce qui explique que, dans le texte, les deux termes soient employés. Pour autant, ils ne sont pas interchangeables et la référence à l'un ou l'autre indique qu'il s'agit de mettre en avant soit la composante biographique (l'immigré est celui qui est né ailleurs), soit la dimension juridique (l'étranger est celui qui n'a pas la nationalité française) de leur condition sociale, sans pour autant sous-estimer les effets de réification, voire de stigmatisation attachés à l'utilisation de ces deux termes (Spire 1999). La participation à l'étude s'est faite, pour moitié, à partir d'une consultation de médecine interne dans un centre hospitalo-universitaire et, pour l'autre moitié, dans le cadre d'une association de prise en charge de personnes africaines malades du sida ; cette double modalité de recrutement, si elle réduit les biais d'échantillonnage, ne les supprime évidemment pas, puisqu'on a affaire, dans les deux cas, à des personnes qui sont en relation avec des institutions, hospitalière et médicale d'un côté, associative et sociale de l'autre. Les entretiens ont été réalisés dans des cadres variés, au domicile de la personne lorsque c'était possible, en d'autres lieux quand elle en manifestait le souhait, mais toujours dans le cadre d'un dispositif d'enquête clairement identifiable en tant que tel; chaque fois que l'accord en était donné, un enregistrement a été fait ; presque toutes les personnes ont été vues à plusieurs reprises et des informations ont ainsi pu être obtenues sur leur devenir. Cette étude s'inscrit dans un programme de recherche sur l'expérience du sida des immigrés et la construction de sa prise en charge par les intervenants médicaux et sociaux en région parisienne, dont les données recueillies, notamment auprès de résidents de foyer et dans diverses institutions de soins, mais aussi auprès des associations d'aide aux immigrés et des services préfectoraux chargés de l'immigration, éclairent les biographies de malades.

L'analyse s'appuiera en effet sur des histoires de vie, et plus particulièrement sur trois d'entre elles (pour lesquelles les noms des personnes et les indications trop précises ont bien sûr été modifiés) illustrant trois modalités du rapport entre condition sociale du malade et condition sociale de l'immigré : le corps légitime, le corps banni, le corps retrouvé. Ces trois figures anthropologiques, loin d'être 
exclusives, entretiennent entre elles une double relation : chronologique, dans la mesure où elles procèdent pour partie de transformations de la réglementation du séjour des étrangers et se succèdent donc non seulement dans le temps collectif, mais également dans l'expérience individuelle; dialectique, dans la mesure où la coexistence de plusieurs référentiels administrativo-juridiques introduit une tension permanente entre elles, soumettant les personnes à des logiques contradictoires. L'accent mis, dans les histoires étudiées, sur le rapport à la loi (au sens strict du juriste comme au sens large de l'anthropologue) peut paraitre sous-estimer les autres dimensions de l'expérience de l'immigré malade ou découler de la forte représentation d'étrangers en situation irrégulière dans l'échantillon étudié. Les options théoriques et méthodologiques qui sous-tendent l'analyse résultent pourtant des données empiriques elles-mêmes : les histoires révèlent à la fois l'importance du statut juridique dans l'expérience des personnes, et notamment dans leur possibilité d'accéder à un traitement, et la variabilité de ce statut dans leur histoire, les faisant passer de la régularité à l'irrégularité en fonction des évolutions de la législation, du contexte idéologique, du marché de l'emploi, des pratiques de l'administration. Si la "question" de l'immigration s'inscrit toujours dans un rapport à l'État (Sayad 1999b), la condition sociale de l'immigré malade s'avère nécessairement être une condition politique.

\section{Le corps légitime}

Peter Igun est nigérian. Il a aujourd'hui trente-neuf ans. Cinquième de huit enfants d'un responsable de la police, il appartient à la bourgeoisie aisée. Après son bac, il obtient une bourse de son gouvernement pour étudier dans une université du Sud des États-Unis, où il découvre le racisme et la ségrégation. Au bout de six années, il obtient un diplôme en pétrochimie et retourne dans son pays. Mais, dans l'intervalle, la situation s'est modifiée et ses espoirs d'une insertion professionnelle sont déçus. L'État, lourdement endetté, ne recrute plus dans l'industrie pétrolière. La vie est devenue chère, les emplois sont maintenant rares et les salaires peu attractifs. Il décide de reprendre ses études pour obtenir un doctorat de sciences, émigrant cette fois vers la France où les frais universitaires lui paraissent plus à la portée de ses ressources. Il change peu après d'orientation et entre dans une école de gestion de laquelle il sort trois ans plus tard avec un diplôme de commerce international. Un stage facilement obtenu dans une agence de courtage allemande se termine par une proposition d'embauche, mais pour un emploi non déclaré qu'il accepte pourtant faute de mieux, dans l'espoir d'une contractualisation ultérieure, une fois son titre de séjour obtenu. Il lui faut donc régulariser sa situation auprès des autorités allemandes, ce qui implique de leur présenter un test récent de sida, pièce constitutive de son dossier.

C'est en faisant cet examen qu'il découvre sa séropositivité, dont il attribue l'origine à la compagne allemande avec laquelle il a vécu plusieurs mois et de qui il a maintenant un enfant. Résolu à rester malgré tout en Allemagne, il falsifie le document du laboratoire biologique et obtient un titre de séjour ainsi qu'une 
autorisation de travail. Mais son employeur refuse de lui donner un contrat et, menacé par lui d'un procès, le met dehors. Lors de son renouvellement de papiers I42 un an plus tard, incapable de présenter une attestation d'emploi, il s'entend signifier son indésirabilité sur le territoire allemand. Sous le coup d'un arrêté de reconduite à la frontière, il se cache et parvient à échapper à plusieurs visites policières à son domicile. Dans ces conditions, il n'est pas question pour lui de se faire suivre médicalement. Après quelques mois ainsi passés à se terrer, il choisit de rentrer clandestinement en France. Il va chez des amis nigérians installés à Paris qui lui avaient proposé un hébergement, mais déchantent quand ils constatent qu'il est sans papiers et sans argent. Deux jours plus tard, leur hospitalité n'est plus de mise et il se retrouve à la rue. Pendant plusieurs semaines, il vit ainsi de mendicité, dormant dans le métro, jusqu'à ce qu’il soit ramassé par les brigades nocturnes et conduit au centre d'hébergement de Nanterre. C'est là que, pendant une année, il passera ses nuits dans un vaste dortoir où il craint de se faire voler et où il redoute surtout, se sachant physiquement fragile, d'être contaminé par ses voisins qu'il entend tousser et cracher. Le jour, il retrouve la rue, puisque le centre le met dehors dès sept heures du matin, mais s'étant fait contrôler à trois reprises, après l'instauration du plan Vigipirate par le ministère de l'Intérieur, et amener à la Préfecture de police, d'où il est cependant chaque fois sorti libre au bout de quelques heures, il n'ose désormais pratiquement plus s'éloigner du centre et circuler dans la ville de peur de se voir reconduire à la frontière.

Cela fait quatre ans qu'il connaît sa séropositivité quand il tombe vraiment malade. Il subit une intervention chirurgicale, les suites opératoires sont émaillées de complications et il passe plusieurs mois à l'hôpital. Transféré dans un service spécialisé, il y reçoit des traitements qui améliorent nettement son état. À sa sortie, il est toujours sans titre de séjour, sans domicile, sans emploi, sans ressources. Il va d'associations en services sociaux pour solliciter quelques aides financières, de centre d'hébergement en armée du salut pour dormir au chaud. Une demande de régularisation " pour raison humanitaire " a été déposée à la préfecture. Après plusieurs mois d'attente, il reçoit une réponse positive et obtient une "autorisation provisoire de séjour pour soins ", assortie d'un droit à exercer un emploi. Les récépissés de trois mois se succèdent et il est désormais muni de " papiers » qui le mettent à l'abri des arrestations. Il consulte régulièrement à l'hôpital et prend sa trithérapie antirétrovirale. Il bénéficie d'un appartement thérapeutique qui lui permet une stabilité résidentielle. Il découvre cependant les difficultés du marché de l'emploi, a fortiori pour un Africain malade n'ayant qu'un titre précaire de séjour. Il n'a pas les trois ans de présence régulière ouvrant droit au revenu minimum d'insertion et demeure donc sans ressources. Quinze ans après son arrivée en France, il survit ainsi, réduit à l'espoir d'une allocation adulte handicapé, évoluant dans un réseau médical et associatif, coupé de ses parents (sa mère est morte sans qu'il puisse assister à son enterrement) et de ses amis (les seuls qui lui restent sont des membres d'associations qui l'ont aidé), sans perspective de retour au pays et sans projet d'insertion dans une société française dont il n'attend plus qu'assistance et où le sida est devenu sa raison sociale. 
D'abord ignorée (jusqu’à la découverte fortuite de son infection), puis négligée (par la force des choses plus que par une volonté d'occultation), la maladie a peu à peu envahi son existence, non seulement par les symptômes physiques et les souffrances psychiques qui en trahissent la présence, mais également par les contraintes des prises médicamenteuses et les obligations de consultations médicales qui la ponctuent. Plus encore, cette affection mortelle est désormais paradoxalement ce qui le fait vivre. C'est elle qui l'avait empêché de retourner au pays, car là-bas, expliquait-il, il serait «mort au bout de quinze jours » faute des traitements et des spécialistes adéquats. Mais c'est aussi en se réclamant d'elle qu'il a obtenu, "pour raison humanitaire ", un titre de séjour et qu'il espère maintenant, son "autorisation de travail » ne lui ayant pas donné accès à l'emploi tant attendu, devenir bénéficiaire du seul minimum social auquel il peut prétendre, celui réservé aux malades. Le sida n'est donc pas seulement ce qui bouleverse le quotidien de son existence, il est ce qui lui donne son prix. C'était à cause de lui qu'il ne pouvait repartir au Nigeria, c'est maintenant grâce à lui qu'il peut demeurer en France: la maladie lui confere un statut juridique, elle lui ouvre droit à une aide sociale. Les travaux portant sur l'expérience des malades atteints d'affections graves (Anderson \& Bury 1988), et singulièrement de sida (Alonzo \& Reynolds 1995), la situent essentiellement dans la sphère privée de la souffrance, même si cette sphère se construit bien entendu dans le rapport avec le monde public du stigmate. Mais, dans le cas des immigrés, et singulièrement des étrangers en situation irrégulière, l'expérience des malades s'élabore dans l'espace public et le rapport à la maladie est un rapport politique au corps.

Pour reprendre la distinction que fait Hannah Arendt $(1983: 49,143)$ entre les deux termes utilisés pour parler de la vie chez Aristote, zồ ou vie physique, biologique, simple parcours de la naissance à la mort, et bios, vie sociale, politique, susceptible aussi d'être racontée comme une biographie, on peut dire que ce qui fait ici le lien entre la condition d'immigré et la condition de malade est la réduction de la bios à la zồe. La bios est en effet ce qui, au sein du règne vivant, distingue l'homme des autres espèces. C'est sa manière à lui d'être au monde, d'exister comme être social et comme animal politique. La condition humaine excède donc la simple zồe, laquelle ne désigne pas seulement, dans le monde grec antique, la vie de l'animal, mais aussi celle de l'esclave et du travailleur, privés par leur activité de la véritable humanité de la bios. Dans le cas de Peter Igun, qu'il faut voir comme l'illustration de la condition plus générale de l'étranger en situation irrégulière, la maladie devient la justification de son existence, son corps physique est ce qui autorise sa présence dans la cité. Comme le constate Giorgio Agamben (1997) à propos du «corps biopolitique de l'Occident", la zồè et la bios se confondent désormais dans sa biographie. Le récit de sa vie est celui de sa maladie, sa seule raison sociale est son sida.

Il y a là bien plus que le constat habituellement fait que la maladie grave tend à envahir l'espace social et mental de la personne qui en est atteinte, constat assurément fondé, au moins à un stade avancé de dégradation du corps (il est en effet probable que l'on surestime souvent la réalité de cet envahissement dans le cas de 
séropositifs asymptomatiques ou peu handicapés par l'infection: d'où l'incompréhension des médecins face aux conduites de dénégation ou d'évitement de 144 leurs patients qui ne mettent pas le virus au cœur de leur existence, notamment lorsqu'ils sont confrontés à toutes sortes d'autres difficultés). Le propos, ici, est de nature radicalement différente. Il est de montrer que, dans ce déplacement de l'expérience du sida depuis l'espace privé vers l'espace public, ce qui se définit, c'est une condition sociale de l'immigré malade. Condition sociale qui s'exprime à la fois comme réalité vécue (la dimension biographique) et comme réalité juridique (la dimension politique). Il suffit pour s'en convaincre d'observer l'évolution de la construction de la subjectivité de Peter Igun autour du sida en fonction des changements des textes législatifs et des pratiques policières vis-à-vis des étrangers au cours des années 90. L'histoire de sa maladie est tout entière inscrite dans l'histoire des politiques européennes de contrôle de l'immigration.

Déjà en Allemagne, lorsqu'en 1992, il découvre sa séropositivité, elle a immédiatement une signification politique. Connu des autorités, le résultat lui interdirait l'accès à un titre de séjour et sa première pensée est de falsifier le document afin de pouvoir obtenir ses papiers et, du moins l'espère-t-il, son contrat de travail. Quand il informe de la teneur du test biologique sa compagne allemande, avec laquelle il est en mauvais termes, elle menace de le dénoncer à la police et de lui faire un procès pour empoisonnement. Dans ces conditions où le péril est partout, il lui parait impossible de voir des médecins ou de contacter des associations car «on ne sait jamais ce qu'ils peuvent faire, s'ils travaillent pour la police ». Il restera donc sans soins et sans aide. Le sida est, dans son histoire, politisé avant d'être médicalisé. Il met en cause sa situation d'immigré plus directement que son état de malade.

Revenu sur le territoire français en 1994 sans titre de séjour, lui qui avait bénéficié d'une carte d'étudiant pendant cinq ans à la fin de la décennie 80 doit se cacher. Son existence s'organise désormais autour d'une double préoccupation de survie quotidienne (où dormir? quoi manger?) et d'insécurité permanente (contrôles d'identité, vols dans la rue). La maladie est alors au second plan de son existence, non seulement parce qu'elle se manifeste peu dans son corps, mais aussi parce qu'elle n'a guère de place dans son histoire. Il la cache aux autres, il ne la soigne pas. Elle ne s'exprime paradoxalement que dans la peur d'être contaminé par des voisins de dortoir dans le centre d'hébergement où il passe ses nuits. Dans cette période, il est un étranger sans papiers et sans domicile plutôt qu'un immigré malade, il relève de l'ordre public plus que de la santé publique.

En 1995, quand il tombe effectivement malade, le sida devient omniprésent dans sa vie quotidienne à cause des symptômes, des souffrances, des inquiétudes, des médicaments, de l'hospitalisation interminable. Pour autant, sa préoccupation principale se concentre sur son titre de séjour. Sa mère est décédée sans qu’il ait pu assister à ses funérailles : «Elle est morte, pour moi, comme une chienne, parce que j'étais pas là pour l'enterrer ». Hanté par le remords, il ne cesse de penser au retour de son propre corps, à sa mort, sur sa terre natale. C'est pour cela qu'il désire tant le titre de séjour : «Il faut que j’aie mes papiers pour pouvoir tra- 
vailler. Je veux pas mourir et être enterré comme un chien. Je veux avoir mes papiers et travailler, et puis comme ça, je vais mettre tout mon argent dans la banque et faire en sorte que, si jamais il m'arrive quelque chose, on me met dans un cercueil et on me transporte en Afrique ». La maladie et la mort, et même, audelà, l'accomplissement de son histoire migratoire, c'est à travers la question du titre de séjour qu'elles prennent toute leur signification. Le sida, désormais présent dans son corps, est ce qui l'empêche de repartir définitivement, car l'absence de moyens thérapeutiques et l'isolement social des patients le condamneraient à une fin rapide et à une agonie pathétique. S'il reste en France, quel que soit le prix à payer en termes d'indignité et d'insécurité, c'est donc maintenant à cause de la maladie.

En 1996, survient l'annonce de sa régularisation dans le cadre de la procédure, instaurée depuis quelques années de l'autorisation de séjour pour soins. Cette mesure, qualifiée d'humanitaire, est une concession accordée à l'esprit des droits de l'homme maintes fois rappelé par la Cour européenne de justice dans une période où se succèdent les lois de plus en plus restrictives sur l'entrée et le séjour des étrangers. La nouvelle catégorie, en pleine expansion numérique, lui permet d'obtenir le statut tant espéré. Précaire, certes, puisqu'il est soumis à réexamen tous les trois mois lors du renouvellement de son récépissé, mais lui permettant de sortir de cette fausse clandestinité, de prétendre à certaines prestations sociales, de chercher un emploi sur un marché où il découvre qu'il ne suffit pas d'avoir des papiers pour pouvoir travailler. Il sait bien cependant que cette autorisation ne lui est accordée que parce que, précisément, son existence est menacée à court terme. Seule la perspective de sa mort physique lui assure la reconnaissance de sa vie politique. L'histoire de son retour à la vie sociale est aussi la chronique de sa mort annoncée.

Parcours remarquable, donc, qui voit s'inverser, à quelques années de distance, le sens même de la maladie. De cette réalité qu'il fallait cacher pour éviter de se voir reconduit à la frontière, elle est devenue une réalité qu'il faut dire pour obtenir le droit de rester sur le territoire. Obstacle irrémédiable, jusqu'à il y a peu, à son entrée ou à son séjour, elle est ce qui justifie la présence de l'étranger. Elle était un secret à garder contre l'État, elle est désormais le lien qui unit à l'État. Au-delà du cas singulier, et si l'on tient compte de l'augmentation rapide des régularisations au titre des soins médicaux dans un contexte où la plupart des autres critères étaient redéfinis de manière restrictive, le statut de la maladie s'est radicalement transformé (Fassin 1999b). Ce qui rendait l'immigré inutile, voire dangereux, est aujourd'hui ce qui le rend légitime. Dans le cas du sida, le corps contaminé, donc potentiellement menaçant, s'est effacé, au moins partiellement, derrière le corps souffrant. La double condition sociale d'immigré malade relève maintenant d'un protocole politique compassionnel.

Dans sa version actuelle, qui s'est substituée, depuis la promulgation de la loi du 11 mai 1998, aux formulations antérieures en termes de "raison humanitaire ", l'ordonnance révisée du 2 novembre 1945 énonce, dans son article 12bis : "La carte de séjour temporaire portant la mention "vie privée et familiale" est 
délivrée de plein droit à l'étranger résidant habituellement en France dont l'état de santé nécessite une prise en charge médicale dont le défaut pourrait entraîner pour lui des conséquences d'une exceptionnelle gravité, sous réserve qu'il ne puisse effectivement bénéficier d'un traitement approprié dans le pays dont il est originaire ». Soumise à l'appréciation d'un médecin inspecteur de la santé dont l'avis, quoique n'ayant qu'une valeur consultative, semble être largement suivi, l'application de ce critère est en principe systématique en cas de sida, conférant au corps malade de l'immigré une légitimité inscrite dans la loi. Ainsi, selon la Direction départementale des Affaires sanitaires et sociales de Seine-Saint-Denis, qui est l'un des deux départements français où se traitent le plus de dossiers d'étrangers sans papiers, le nombre d'autorisations provisoires de séjour pour soins a été multiplié par quatre au cours des années 90. De même, selon la Direction des Libertés publiques du ministère de l'Intérieur, lors de la campagne de régularisation de 1997-1998, plus de trois mille personnes, soit environ un dossier sur dix pour lesquels un titre de séjour a été délivré, ont bénéficié de cette mesure au titre de la maladie.

À s'en tenir au texte de ces dispositions législatives, il ne devrait d'ailleurs plus y avoir d'étrangers en situation irrégulière souffrant de sida. Tel n'est toutefois pas le cas, notamment à cause de la mention, au début de l'article cité, qu'une carte de séjour temporaire sera donnée, conformément aux conditions indiquées, à l'étranger demandeur «sauf si sa présence constitue une menace pour l'ordre public». Le second récit illustre ainsi les hiérarchies établies dans l'ordre de la légitimité pour administrer les immigrés malades.

\section{Le corps banni}

Karim El Mahdi est marocain. Il vit en France depuis qu'il a l'âge de douze ans. Son père était venu le premier, travailleur immigré dans le bâtiment à une époque où, sur le marché de l'emploi, la main-d'œuvre étrangère, docile et peu coûteuse, était particulièrement prisée. Une fois sa situation économique et juridique convenablement établie, il fait venir sa femme et ses quatre enfants. On est en 1972. L'immigration commence certes à être contrôlée, mais le droit de vivre en famille apparaît alors à l'ensemble du monde politique, tous partis confondus, comme une évidence morale. L'obtention des titres de séjour se fait sans problème dans le cadre d'un regroupement familial régularisé a posteriori, comme il sera habituel jusqu'au milieu des années 80 . La famille réside dans l'une des grandes cités dites en difficulté de la région parisienne. Ladolescent n’a pas de bons résultats au collège qu'il quitte en classe de quatrième pour faire un stage de tourneur. Au terme de cet apprentissage, il ne trouve cependant pas d'emploi. Désœuvré, il passe ses journées avec des jeunes de son âge partageant une situation similaire à la sienne. Il commence à se droguer, d'abord avec du cannabis, puis à l'héroïne. Il fait plusieurs séjours en prison pour des affaires de stupéfiants. Son père lui obtient un contrat de manœuvre sur son chantier, mais le contrat est bientôt interrompu. Il travaille quelques mois en intérim comme manuten- 
tionnaire, se fait ensuite embaucher comme coursier mais, sa moto étant hors d'usage, il renonce à cette activité. On est en 1988, il ne trouvera plus jamais d'emploi. Peu avant, il avait découvert qu'il était séropositif et avait décidé d'arrêter de se droguer, mais s'était mis à boire.

À seize ans, on lui avait suggéré de demander la nationalité française. Il ne l'avait pas fait et avait bénéficié, à sa majorité, d'une "carte de dix ans " assortie d'une autorisation de travail. Pendant près d'une décennie, il avait ainsi été en situation régulière, mais, à la suite de ses démêlés avec la justice, une interdiction de territoire avait été prononcée et son titre de séjour lui avait été retiré. La mesure ne pouvait toutefois en principe pas être exécutée, car vivant en concubinage avec une femme française depuis la fin des années 70 , il en avait eu quatre enfants qu'il avait reconnus et qui tous étaient français. Il entrait donc dans la catégorie des «non régularisables non expulsables" que les associations de défense des immigrés dénonçaient alors. Cependant, le flou qui entourait cette catégorie et la méconnaissance que le jeune Marocain avait de ses droits, les mesures abusives d'éloignement qui avaient parfois eu lieu dans des cas semblables et les dénonciations effectuées auprès de la préfecture par des agents municipaux, le rendaient particulièrement réticent à l'égard de toutes les institutions, tant d'aide sociale que de soins médicaux. En 1994, une tuberculose pulmonaire le conduit cependant à l'hôpital à un stade déjà avancé de l'infection. Il se fait enregistrer sous le nom de son frère pour bénéficier de son assurance maladie. Découverte par les services administratifs, "l'usurpation d'identité " conduit à la suspension de la "prise en charge à cent pour cent» et surtout à une suspicion diffuse à son encontre. Ses médecins sont persuadés qu'il ne prend pas son traitement et qu'il jette les médicaments. L'un d'eux note même dans le dossier médical qu'il est "polygame". À sa sortie de l'hôpital, il ne sera pas suivi. En quelques mois, son état physique se dégrade considérablement.

Lors du premier entretien, qui se déroule à son domicile, il est très affaibli et amaigri. Il vit, avec sa compagne et ses quatre enfants, au cœur de la cité où il a passé son enfance, dans un appartement de trois pièces squatté dont les volets sont abaissés pour que la présence de la famille ne soit pas repérée de l'extérieur. Crainte qui n'est du reste pas sans fondement, puisque la municipalité de la ville dans laquelle il réside est connue pour ses dénonciations d'étrangers en situation irrégulière à la préfecture, à l'occasion notamment de demandes d'aide médicale que ces derniers lui adressent. Les conditions économiques du ménage sont particulièrement précaires. Étant donné que lui n'est pas censé être sur le territoire français et puisqu'ils n'ont pas d'adresse officielle, ils ne bénéficient d'aucun des minima sociaux et survivent grâce à des emplois temporaires qu'obtient la femme et à des aides ponctuelles que leur consent le service social départemental. Malgré ses réticences initiales, le malade finira par accepter une hospitalisation dans un autre établissement. Trop tard, cependant. Une succession de complications du sida aboutira à son décès. Il était âgé de trente-six ans.

Cette histoire pourrait certes être lue comme une série d'événements dramatiques relevant d'enchaînements circonstanciels malheureux : occasion manquée 
de l'acquisition de la nationalité française qui l'aurait définitivement mis à l'abri du risque d'exclusion juridique; découverte du chômage dès la sortie de son apprentissage qui va l'exposer au désœuvrement et à la déviance ; rencontre avec la drogue qui le conduit en prison et constitue très probablement la source de sa contamination; recours à une fausse identité lors de sa première hospitalisation qui compromet les relations avec les médecins; et l'on pourrait même ajouter, aggravation de sa maladie dans une période de durcissement répressif à l'encontre des étrangers en situation irrégulière - ce qui accroît leur insécurité et favorise les dénonciations, autrement dit rend plus difficile l'accès aux soins. Une telle lecture laisserait pourtant échapper les réalités structurelles, à la fois sociales et politiques, qui sous-tendent cette histoire et en rendent les enchaînements, à première vue fortuits, aisément explicables au contraire. Il suffirait pour s'en convaincre de reprendre une série de biographies de jeunes adultes étrangers, principalement maghrébins, malades du sida: on y retrouverait les mêmes arrivées en France dans l'enfance ou l'adolescence, les mêmes expériences du chômage et de la drogue dans les cités, et pour ceux qui n'auraient pas pu ou voulu acquérir la nationalité française, les mêmes condamnations simultanées à la prison et à l'interdiction de territoire, aboutissant aux mêmes situations inextricables en regard desquelles les maladies les plus graves ne font pas contrepoids devant les juges: tout au plus rendent-elles les mesures d'éloignement inapplicables, du moins en principe car une série d'expulsions de patients atteints d'affections mortelles ont été dénoncées au cours des dernières années.

Même partielles, les statistiques de l'Institut de veille sanitaire ne laissent guère de doute sur l'exemplarité de la biographie présentée (Lot 2000). Près de la moitié des malades du sida ressortissants d'Afrique du Nord se sont contaminés par l'usage de drogues, soit deux fois plus que pour les Français. Si l'on tient compte du fait que l'incidence globale de l'infection est le double chez les premiers par rapport aux seconds, c'est dire que les hommes d'Afrique du Nord sont quatre fois plus exposés que les Français à la transmission du sida par injection de drogues. Et l'on peut ajouter que la proportion de sans emploi et la part des ouvriers parmi les premiers sont également deux fois plus importantes que celles observées chez les seconds. De plus, même si l'on ne dispose pas de statistiques à ce sujet, la plupart des malades du sida contaminés dans le cadre de pratiques toxicomaniaques ont eu maille à partir avec la justice et nombre d'entre eux sont sous le coup d'une mesure judiciaire d'interdiction de territoire, provisoire ou parfois définitive, pour trafic ou vol.

Bien que son histoire soit française pour les deux tiers, correspondant à son adolescence, à sa jeunesse et à sa brève vie d'adulte, Karim El Mahdi est bien étranger et immigré. Étranger, en ce que, lui-même marocain de parents marocains, il n'a pas pris la nationalité française lorsque cela lui était possible. Immigré, en ce qu'il est né au Maroc et y a vécu enfant, ne venant résider en France qu'au début de son adolescence. Mais au-delà de cette définition littérale, juridique et biographique, de son état, c'est sa condition sociale qui fait de lui, à ses propres yeux comme pour les autres, un étranger et un immigré. Condition sans histoire, faudrait-il dire, car il est un étranger qui ne connaît pratiquement 
pas le pays dont il a la nationalité et un immigré qui se souvient à peine du lieu où il est né. Condition qui n'a de sens que dans une économie politique de l'immigration où la $z \hat{o} e ̀$ du travailleur est sa seule justification, où la bios du citoyen se trouve réduite à la portion congrue. Ainsi le père a-t-il pu trouver sa place en France, et celle de sa famille, par son activité dans la production, tout en demeurant dans les espaces marginaux de la cité civique. À la seconde génération, quand le travail est devenu rare, quand la perte de citoyenneté est accomplie avec l'exclusion juridique, l'immigré n'a plus de raison d'être. Il ressortit même au régime d'exception que représente la double condamnation à la prison et à l'exil. Il n'est plus qu'une menace à l'ordre public justifiant sa mise hors-la-loi. Plus encore que des « inutiles au monde» dont parle Robert Castel (1995 : 16) pour désigner ces hommes et ces femmes sans emploi ni perspective d'emploi, on a affaire à des « indésirables » pour lesquels, comme le dit Hannah Arendt (1982: 261), l'État peut se permettre "quelques actes illégaux", ou tout au moins illégitimes au regard de l'esprit des lois. Avant d'en arriver à cette extrémité, l'immigré a cependant parcouru une trajectoire que l'on peut assimiler à la carrière dont parle Howard Becker (1985) pour analyser la déviance. Carrière en l'occurrence sociale tout autant que politique.

Durant son adolescence, Karim El Mahdi fait l'expérience de la ségrégation et de l'inégalité liées à l'immigration. Sa famille réside dans l'une des plus grandes cités de la région parisienne. En situation d'échec scolaire, il quitte le collège à l'âge du minimum légal de fin de scolarité sans être orienté vers une filière professionnelle "parce qu'on a vu que ça ne l'intéressait pas »: il sera donc comme son père ouvrier non qualifié. Il fait un stage de tourneur, mais ne trouve pas d'emploi car «le niveau de qualification exigé par les entreprises était trop élevé ": il est d'emblée au chômage sans jamais avoir travaillé. Un peu plus tard, les emplois de manœuvre, de manutentionnaire et de coursier qu'il exerce, sur des contrats précaires, sont entrecoupés de longues périodes d'inactivité, au cours desquelles il découvre la drogue et la délinquance. Durant cette période, s’il ne demande pas la nationalité française, c'est qu'une telle sollicitation ne fait guère sens pour lui et ce pour deux raisons en partie contradictoires : d'une part, résidant en France avec des parents qui s'y sont définitivement installés et des frères plus jeunes qui sont Français, il ne perçoit pas la menace d'une remise en cause de la légitimité et a fortiori de la légalité de sa présence sur le territoire français ; mais, d'autre part, confronté à ce qu'il perçoit comme un rejet de la ville, de l'école et du travail, il ne voit pas non plus la nécessité d'effectuer cet acte d'allégeance à une République de laquelle il se sent exclu. Pour l'exprimer autrement, même s'il ne se sent pas vraiment étranger, il s'entend rappeler sans cesse par la société sa différence en tant qu'immigré. Plus banalement, ces deux raisons trouvent leur expression dans le fait que la question de l'acquisition de la nationalité française ne l'intéresse pas: "Je me rendais pas compte, j'ai même pas réfléchi sur le coup, et puis voilà, maintenant je regrette ». S'il manifeste ce regret, c'est que deux événements sont survenus, à peu de temps d'intervalle, dans sa vie : l'interdiction définitive du territoire français et la découverte de sa séropositivité. 
La désignation de la déviance avait d'abord suivi le cours assez ordinaire des interpellations, des incarcérations, des inscriptions au casier judiciaire: «À cette époque, en prison, j'entrais et je ressortais, j'entrais et je ressortais sans arrêt ». Bien plus que ces épisodes qui finissent par lui apparaitre comme une nouvelle normalité, la véritable rupture, c'est la sanction prononcée lui interdisant de rester en France : lorsqu'elle tombe, il est déjà avec sa compagne et père de deux enfants français. Aboutissement pour lui totalement inattendu de sa carrière déviante, elle vient le rappeler à sa condition d'immigré mais aussi d'étranger, c'est-à-dire de toujours potentiellement illégitime et désormais irrémédiablement illégal (Palidda 1999). C'est la même année que tombe l'annonce de son infection par le virus du sida. Tout comme la mesure judiciaire prise à son encontre, elle semble marquer le terme de son parcours. La maladie apparaît comme le résultat d'une série de circonstances sinon prévisibles a priori, du moins interprétables a posteriori quand on sait que le taux de séroprévalence parmi les héroïnomanes était estimé à près de quarante pour cent dans cette période où la politique de réduction des risques liés à la toxicomanie venait tout juste d'être instituée, mais n'était pas encore appliquée (Bergeron 1999). Interdiction du territoire et découverte de l'infection marquent la double clôture de son histoire.

À la différence du cas précédent, le sida ne vient pas proposer une issue, aussi dramatique soit-elle, à l'épreuve de l'irrégularité : il en est au contraire la circonstance aggravante. Le banni peut d'autant moins se résoudre à l'exil qu'il se sait malade d'une maladie qui, dans le pays vers lequel on compte le reconduire, ne pourrait être soignée mais surtout l'exclurait d'avantage. Il est conduit à se terrer, renonçant à ses droits qu'il ignore et que personne ne lui rappelle, à commencer par la possibilité de demander le relèvement de la décision sous couvert d'une assignation à résidence, mais aussi l'accès à une aide médicale pour obtention de ses soins. Il reste donc sans se soigner, du moins jusqu'à la dernière extrémité : une tuberculose qui est diagnostiquée à un stade avancé de son développement, la première fois, et des complications multiples dans un contexte clinique de dénutrition qui s'avéreront fatales, la seconde fois. Dans cet au-delà de la loi, qui est aussi un au-delà du droit, la logique humanitaire n’a plus sa place.

Cette figure du corps indésirable, radicale jusque dans la suppression de la vie, n'est pas sans rappeler l'étrange "homo sacer» du droit romain archaïque dont Giorgio Agamben $(1997: 81,121)$ a fait un élément central de sa réflexion sur le monde contemporain. On sait qu'il s'agit de «celui que le peuple a jugé pour crime : il n'est pas permis de le sacrifier, mais celui qui le tue ne sera pas condamné pour homicide». Sans revenir sur les exégèses nombreuses de cette réalité plus anthropologique que juridique, on peut suivre le philosophe italien lorsqu'il invite à dépasser la discussion philologique et historiographique pour repérer les formes contemporaines de «l'expulsion hors de la communauté » qui pourrait s'avérer de puissants révélateurs du sens de la souveraineté : «C'est cette structure de ban que l'on doit apprendre à reconnaitre dans les relations politiques et les espaces publics où nous vivons encore ». En l'occurrence, si personne ne tue Karim El Mahdi, la société le condamne cependant bien à un enfermement dans sa condition de 
malade dont la seule issue est la mort, et ce non tant pour les délits qu'il a commis qu'en raison surtout de sa condition d'immigré rabattue sur un statut d'étranger hors-la-loi : s'il était Français, en effet, les mêmes délits ne conduiraient évidemment qu'à des peines d'emprisonnement, sans remettre en cause son droit à se trouver sur le sol français et donc ses possibilités de survivre. Certes, l'hôpital ne lui refuse pas les soins et l'assistante sociale souhaite l'aider, mais le dispositif répressif dans lequel il se trouve pris est tel qu'il a peur de recourir à quelque institution que ce soit et que même les services sociaux affirment hésiter à solliciter pour lui une aide médicale par peur, justifiée dans ce cas précis, de dénonciations à la préfecture (Fassin 1996b). La mise au ban de la société de l'étranger délinquant opère bien ici comme une condamnation à mort. Ce corps indésirable est celui d'une vie qui n'est plus digne d'être vécue. À la bios déjà abolie de manière relative dans la relégation sociale, puis absolue dans l'exclusion juridique s'ajoute désormais la négation de la zôé dans le renoncement à soigner la maladie et à alimenter l'organisme. Si l'interdit de séjour atteint par une affection grave peut encore, en principe, recevoir des soins et de la nourriture, les conditions sociales de son existence ne le permettent de fait souvent plus.

Quelles que soient cependant les logiques d'anéantissement qui sont à l'œuvre dans cette figure extrême - mais relativement fréquente -, elles ne sauraient empêcher la permanence ou parfois même le développement de logiques contraires. Pour le dire plus simplement, à la mise à mort sociale et physique répond une volonté de vivre à la fois politiquement et biologiquement. Dans le cas de Karim El Mahdi, cette volonté est certes ténue : on peut à cet égard considérer comme une forme de résistance politique le récit de son histoire, notamment parce qu’il lui permet d'énoncer publiquement sa vérité ("une fois, le médecin est venu me voir le matin, elle m’a dit: je suis sûr que vous jetez vos médicaments ; mais c'est pas vrai, je les prenais tous ", affirme-t-il, et ce détail apparent n'en est un ni pour les soignants qui l'ont disqualifié par leur méfiance, ni pour lui qui réclame cette dernière parcelle de justice à son égard); on peut de même considérer comme une forme de résistance biologique l'arrêt de la toxicomanie une fois la séropositivité découverte ou l'acceptation des traitements dès lors qu'on le rassure sur l'absence de risque de dénonciation (« je me traite à cause des gosses, c'est trop sérieux »). Oblitérées par la rigueur de la loi, les deux formes de vie, bios et zốé, ne sont donc pas totalement anéanties dans les interstices de la société où il se débat encore. Toutefois, de cette réaction vitale face à la double condamnation par la maladie et par la justice, il est heureusement des expressions moins dramatiques. C'est ce que montre la troisième étude de cas.

\section{Le corps retrouvé}

Sophie Mugangu a grandi à Kinshasa. Deuxième d'une famille de onze enfants de classe moyenne, elle est allée au collège, puis a fait des études d'infirmière et commencé à travailler à l'hôpital. Elle s'est mariée et a eu deux filles, mais son mari buvait et la battait. Ils se sont séparés et quelque temps après, il est mort. Elle 
décide alors de refaire sa vie en Europe en confiant ses enfants à ses parents. Elle a vingt-cinq ans. Elle vient en Belgique avec un visa touristique pour y rejoindre une compatriote dont elle garde les enfants. À la suite d'un contrôle alors que son visa a expiré, ses papiers lui sont confisqués et sa patronne se débarrasse d'elle. Se trouvant seule, elle part pour la Suisse où elle dépose une demande d'asile politique, ce qui lui permet d'obtenir un titre de séjour provisoire et de travailler dans un hôpital, mais comme manutentionnaire dans les cuisines. Déboutée, elle traverse la frontière et s'installe en France où elle sollicite de la même manière l'asile politique; cette fois, le récépissé lui ouvre droit à une aide financière, mais pas à un emploi. À Lyon où elle réside maintenant, elle rencontre un Camerounais et ils projettent de se marier. Pour elle, c'est la perspective d'obtenir un statut juridique et en même temps stabiliser sa situation économique. Lors de la visite médicale prénuptiale, on lui fait le test du sida et on découvre sa séropositivité. Son fiancé, lui, est séronégatif. Elle garde le secret vis-à-vis de lui, mais craignant sa réaction de rejet s'il l'apprend, elle prend les devants et s'enfuit.

À Paris, où elle n'a pas de famille ni d'amis, elle passe plusieurs semaines à la rue, dormant dans des appartements inoccupés, des escaliers d'immeuble, des hangars de supermarché ou parfois dans le métro. C'est là qu'elle fait la connaissance d'un Zaïrois duquel elle est, peu après, enceinte et qui l'abandonne alors. Contre l'avis des médecins, elle décide de mener la grossesse à son terme, s'en remettant à Dieu et à l'antirétroviral qui lui a été prescrit. Aidée par une association spécialisée dans la prise en charge des femmes malades du sida, elle obtient une chambre et vit en faisant des ménages sans être déclarée. Les journées sont rythmées par la prise du médicament toutes les quatre heures. Au moment de la naissance, on la sépare de son enfant que l'on isole en lui disant qu'il risque de contaminer les autres nouveau-nés. Il n'est pourtant pas infecté. Pas plus que le second enfant qu'elle a deux ans plus tard. À chaque grossesse, pourtant, elle doit subir les remarques désobligeantes de membres du personnel hospitalier qui ne comprennent pas qu'elle soit de nouveau enceinte alors qu'elle se sait infectée. Lors du second accouchement, une sage-femme lui dit qu'elle n'aura pas de péridurale pour que la douleur lui fasse passer l'envie d'avoir d'autres enfants. Au moment du premier entretien, elle est pourtant enceinte pour la cinquième fois.

Toujours sans titre de séjour, elle est parvenue à trouver un emploi comme femme de chambre dans un hôtel en utilisant les papiers d'une amie. Peu après, par l'intermédiaire d'une association dont il est l'un des donateurs, elle entre en contact avec un homme dont le frère est mort du sida et qui met à sa disposition un studio. Elle s'y installe avec les deux enfants qui vivent avec elle. Grâce à l'assistante sociale d'une autre association, elle fait les démarches auprès de la préfecture et obtient une autorisation provisoire de séjour pour soins lui ouvrant droit au travail. La voilà désormais en situation régulière et en mesure de chercher un emploi à son nom. Une aide de la mairie lui permet de bénéficier de la gratuité de la cantine pour son fils aîné. Le soutien d'une association lui assure la garde de son cadet pendant qu'elle travaille. Quant à ses examens et ses traitements, elle n'en supporte pas les frais, bénéficiant de la carte Paris Santé. Ses amies, c’est dans les 
associations de prise en charge des malades du sida qu'elle les a connues. En revanche, elle a rompu avec la plupart de ses compatriotes ou parents par peur des indiscrétions sur son état clinique. Ses deux fils, qui ont deux et quatre ans, lui sont un grand réconfort. Mais une convocation chez le juge pour enfants lui fait craindre qu'on ne les lui retire à cause de la maladie ou de ses habitudes, penset-elle. Les travailleurs sociaux trouvent en effet qu'elle sort trop et voit trop d'hommes. Quatre ans après la découverte de la maladie et malgré les épreuves du quotidien, elle fait face à toutes les vicissitudes de son existence. Elle est maintenant âgée de trente-quatre ans. Lorsqu’on lui avait annoncé sa séropositivité, elle s'était donné dix années à vivre. Il lui en reste quatre aujourd'hui.

La condition d'immigré et la condition de malade ne s'imposent pas à des agents passifs. Bien au contraire, les politiques de l'immigration et les contraintes de la maladie donnent lieu à ce que Michel de Certeau (1980:21) appelle des «tactiques " par lesquelles les dominés se réapproprient, au moins partiellement ou transitoirement, les règles du jeu social : «La tactique n'a pour lieu que celui de l'autre. Du fait de son non-lieu, la tactique dépend du temps, vigilante à y "saisir au vol" des possibilités de profit. Ce qu'elle gagne, elle ne le garde pas. Il lui faut constamment jouer avec les événements pour en faire des "occasions". Sans cesse le faible doit tirer parti de forces qui lui sont étrangères ". De manière exemplaire, la vie de Sophie Mugangu se construit à travers la mise en ouvre de ces tactiques. Face à la double loi, celle de l'État et celle du sida, qui s'impose à son corps, elle développe des "formes d'ajustement» en mobilisant des "ressources" (Pollak 1990 : 293) qui lui permettent de faire avec, non seulement en s'adaptant aux événements, mais aussi en les retournant à son avantage.

Venir en Europe résulte d'un projet migratoire explicite de "refaire sa vie». Projet dont le caractère définitif s'avère d'autant plus manifeste a posteriori quand on constate qu'elle a, au cours des seize années de son séjour hors de son pays, rompu pratiquement tous les liens avec ses parents, ses amis et même ses deux filles. Certes, les circonstances tant de l'irrégularité de sa présence que de la découverte de l'infection ne lui ont guère laissé de chances de revenir en arrière, ou même de rester à mi-chemin de deux histoires en retournant à Kinshasa, ne serait-ce que pour des périodes brèves comme le font souvent les femmes africaines dans sa situation (Quiminal 1991) : sans titre de séjour, le risque de ne pas pouvoir rentrer en France était trop élevé et, compte tenu de la maladie, mettait sa vie trop en danger ; de surcroît, l'existence du virus apparaissait comme une donnée trop stigmatisante dans son milieu d'origine pour qu'elle envisage de s'exposer à des questions ou de se laisser aller à des confidences. Pourtant, la rupture biographique semble consommée dès sa décision de partir : «J'ai fait mes deux enfants et puis j’ai changé d'idée. J'ai dit : je change de pays. Je suis venue en Europe ». Le raccourci de la formulation ne dit certes rien des raisons profondes de son départ, mais il exprime le caractère radical d'un changement d'existence qui suppose le renoncement simultané à une histoire, une famille, un métier.

Cette volonté d'une autre vie se heurte à la réalité des législations européennes sur l'entrée et le séjour des étrangers. Elle parvient toutefois à les contourner, au 
moins en partie. Pour sa première tentative, en Belgique, elle a recours au visa touristique pour pénétrer en Europe, mais se fait prendre alors qu'elle travaille I54 illégalement chez une compatriote qui l'a fait venir pour occuper cet emploi. Pour la seconde et la troisième, en Suisse puis en France, mieux avisée et conseillée, elle fait des demandes d'asile politique qui, si elles n'ont quasiment aucune chance d'aboutir à un accord au regard des critères de l'Office français des réfugiés et apatrides pour les sollicitants zaïrois, permettent au moins l'obtention d'un titre provisoire autorisant le travail dans le premier pays et des aides dans le second. Ainsi, de récépissé en récépissé, de recours en recours, réussit-elle à se maintenir plusieurs années régulièrement sur le territoire européen, ne quittant la Suisse qu'au moment où les diverses procédures possibles semblent avoir été épuisées et se mettant alors en quête d'un mari bénéficiaire d'une "carte de dix ans " dès son arrivée en France, dans l'espoir d'obtenir elle aussi un titre de séjour définitif: "Le Camerounais, c'est vrai, je l'avais accepté parce que je voulais les papiers. Bon, lui aussi était malin. Il me dit : avant de te donner les papiers, il faut m'épouser et me faire des enfants ». Seule la découverte du sida, avec les perspectives d'indiscrétion et de répudiation qu'elle entrevoit, la fait renoncer à son projet et la met brutalement à la rue. Cependant, même réduite à cette solution extrême, elle la présente encore comme un choix : "Je préfere aller seule. Quand j'ai fui, j'ai même dormi dehors, ici à Paris pendant l'hiver». Et c'est dans le métro qu'elle rencontre le père de son troisième enfant.

Loin de la faire sombrer dans le désespoir ou la résignation, les risques encourus pour cette grossesse menée alors qu'elle se sait infectée la conduisent à développer de nouvelles formes de résistance. Alors que le médecin lui conseille un avortement thérapeutique, elle lui répond : "Je veux voir la puissance de Dieu ", tout en se mettant à la recherche d'un service hospitalier où l'on pourra la suivre et la traiter pendant sa grossesse. Sa confiance dans l'avenir se trouve du reste confortée par l'absence de contamination des deux enfants auxquels elle a donné naissance alors qu'elle est elle-même porteuse du virus, mais aussi, de manière moins anecdotique qu'il n'y paraît, par la chance que le premier d'entre eux lui apporte dans les jeux de hasard, notamment au loto et au tiercé : "L'enfant, il m'a donné beaucoup d'argent ». Elle se soumet à toutes les contraintes de son traitement, y compris aux effets secondaires de sa trithérapie, et surveille ses indicateurs biologiques, dont elle connaît précisément la valeur et la signification. Sa philosophie semble tenir dans un épicurisme sobre où la jouissance a certes sa place (et elle raconte avec plaisir ses aventures amoureuses), mais qui repose avant tout sur un évitement des souffrances physiques et psychiques (et lors de ses grossesses, elle dit ne pas même envisager que ses enfants puissent être infectés). "Vivre dans cette maladie, bon c'est pas dit qu'on va mourir aujourd'hui. Il faut lutter, il faut se battre ! Il faut pas mettre ça dans la tête. Moi, j'ai dit que j'ai encore dix ans. Bon, maintenant c'est six ans (rire). Six ans... J'attends encore quatre ans ». Probablement ces déclarations ne doivent-elles pas être prises pour argent comptant: l'optimisme affiché ne signifie pas nécessairement une véritable sérénité. Il n’en demeure pas moins qu'au-delà des mots, tout témoigne, 
dans son histoire, de sa capacité à faire face aux épreuves. Le sida est pourtant à l'origine d'une double rupture dans sa vie de femme immigrée.

Rupture biologique, tout d'abord. Son existence quotidienne s'inscrit désormais dans le cadre de la maladie, de ses médicaments, de ses indicateurs. L'infection fait de son corps un corps souillé : "Quelqu'un qui est infecté, tu n'es pas propre, tu as le sang sale ». Face à la menace vitale que représente le sida, la médicalisation devient pour elle la condition, au double sens du mot, de son existence : «Le traitement, ça fait quatre ans, je prends ça et je vis ». Durant les grossesses, l'antirétroviral doit être absorbé "toutes les quatre heures jusqu'à zéro heure ", ce qui lui semble "très très dur ", mais lui permet paradoxalement de "vivre comme une femme négative qui est enceinte ». À chaque consultation, elle est informée des variations des résultats des examens qui prennent ainsi place dans la représentation de son état physique, lui faisant dire que "les T4 sont un peu bas parce que je suis enceinte, ils sont à 125 alors que c'était remonté à 300 », se rassurant toutefois car «si j'accouche, peut-être ça va en remontant, on me donne aussi d'autres médicaments». Mais les produits qu'elle prend contraignent et restreignent son espace de vie. La régularité des prises et l'effort pour les dissimuler l'ont amenée à réduire ses échanges avec le monde extérieur et à se confiner chez elle. Même ainsi, elle n'est pas à l'abri d'une mésaventure et elle raconte l'expérience éprouvante qu'a été pour elle le fait qu'une de ses amies, au cours d'une visite chez elle, ait découvert et dérobé ses médicaments, révélant publiquement son sida.

Rupture sociale, ensuite. Dans la mesure où la maladie est source non seulement de stigmatisation, mais aussi d'interprétations morales (sur la conduite antérieure de la personne infectée, et la liberté de sa sexualité lui a d'ailleurs valu des reproches) et d'accusations potentielles (sur les partenaires contaminés, à commencer par son premier mari décédé), il n'est pas question d'en parler à l'entourage. En l'occurrence, aucun membre de la famille, aucun ami congolais n'a été mis dans la confidence: "J'ai pas confiance en personne. Je dis jamais à personne. C'est ma vie propre». Peu à peu, elle s'est coupée de son milieu d'origine, éloignement qui a du reste été précipité par la suspicion et la jalousie qu’a suscitées, dans son entourage, l'obtention d'un appartement fourni par une association. Un nouveau réseau s'est constitué autour d'elle, entièrement lié à sa maladie et à l'inscription de celle-ci dans son histoire de femme immigrée : ce sont les médecins, les travailleurs sociaux, les membres d'associations de prise en charge des malades du sida, dont certaines plus particulièrement impliquées dans l'aide aux étrangers. Toutes ses amies sont aujourd'hui des femmes qu'elle a rencontrées dans ces lieux entièrement dévolus à la maladie. Elles partagent leurs expériences de l'infection, des traitements, des bonnes adresses. Quant aux hommes, elle les divise en deux catégories : ceux, "africains", dont elle fait la connaissance en dehors des associations et auxquels elle ne dit pas son statut sérologique, leur demandant seulement d'utiliser un préservatif; ceux, "européens ", qu'elle rencontre dans ces lieux et avec lesquels la question du virus peut 
être évoquée. Mais elle reconnait que l'infection a changé sa sexualité : «Moi, je me colle beaucoup, j'aime bien les hommes (rire). Mais cette maladie-là, ça me bloque, ça empêche d'aimer ». Sa vie sentimentale se partage désormais entre des aventures d'une nuit et le rêve impossible d'un grand amour.

L'histoire de Sophie Mugangu se donne ainsi à lire comme une série de petits arrangements avec la vie, cette vie vécue dans la double condition de malade et d'immigrée. Vie biologique à laquelle elle doit plier son corps tout en se réappropriant le langage et les outils de la médecine. Vie sociale qui lui est imposée mais qu'elle réorganise en fonction de la nouvelle donne. Cette redéfinition de son existence, à la fois comme zôé et comme bios, correspond à ce régime particulier de "biosocialité " dont parle Paul Rabinow (1996) à propos des personnes dont la vie comme être biologique et comme être social se trouve très largement déterminée par l'existence d'une maladie ou même d'un risque génétique. Dans le cas présent, il s'agit en effet plus que d'une attitude pragmatique consistant à simplement faire avec: il y va aussi d'une subjectivation inscrite dans le corps comme un vivre avec qu'il faut prendre au sens fort d'une restructuration complète de l'existence autour de la maladie sous contrainte de la précarité économique et juridique de l'immigrée.

Que, dans le récit présenté, cette figure du corps retrouvé, à la différence des deux précédentes, concerne une femme ne doit pas étonner. Même si certains traits peuvent exister aussi dans des biographies d'hommes et même si, à l'inverse, les deux autres figures peuvent aussi être féminines, l'enquête révèle une double raison à cette différenciation sexuelle des conditions. Premièrement, les femmes sont beaucoup moins confrontées aux pratiques répressives de l'État. D'une part, elles semblent peu concernées par les contrôles d'identité et les reconduites à la frontière; elles-mêmes en sont conscientes, déclarant volontiers qu'elles circulent assez librement en ville; dans les rares récits incluant une arrestation, la remise en liberté a été immédiate; moins directement menacées, elles vivent donc moins fortement le changement de statut par lequel le corps souffrant se trouve investi d'une nouvelle légitimité politique, caractéristique de la première figure. D'autre part, elles sont peu exposées à la perspective de l'interdiction du territoire, laquelle concerne principalement les actes délictueux ayant fait l'objet d'une condamnation et les refus d'embarquement lors des reconduites à la frontière; par rapport à la première situation, elles sont moins impliquées dans les conduites toxicomaniaques avec leur double conséquence potentielle en termes d'infection et de pénalisation; par rapport à la deuxième, elles font moins l'objet de procédures de reconduite à la frontière; ainsi apparaissent-elles moins prisonnières de cette exclusion juridique du corps malade, caractéristique de la seconde figure. Deuxièmement, les femmes sont beaucoup plus que les hommes confinées dans la sphère privée. Loin de correspondre à une vision naturalisée ou banalisée d'une condition féminine, cette réalité procède là encore d'une double assignation par l'immigration et la maladie. D'un côté, les femmes, souvent venues hors du cadre réglementaire, tombent alors sous la dépendance d'un conjoint en situation régulière qui dispose d'un moyen 
efficace de la garder au domicile et de la cantonner dans des tâches domestiques. De l'autre, lorsqu'elles sont infectées, la question de la reproduction et de la transmission possible à leur enfant devient un élément essentiel de leur expérience de la maladie et détermine, tant dans le projet de procréation qu'à travers la surveillance ou le traitement des enfants, leur existence quotidienne. Ce retrait relatif d'un espace public, au demeurant moins dangereux, permet ainsi aux femmes de développer au quotidien les tactiques de cette troisième figure, dont elles n'ont toutefois pas l'apanage.

\section{Les biopolitiques de l'immigration}

Corps légitime, corps banni et corps retrouvé: ces trois figures anthropologiques révèlent trois modalités de mise en œuvre de la double condition d'immigré et de malade. Dans le premier cas, le corps de l'étranger indésirable trouve sa reconnaissance en tant qu'il est affecté par le sida. La condition d'immigré est juridiquement réinterprétée à travers la condition de malade. La raison humanitaire prévaut. La vie sociale s'enracine dans la vie biologique. Dans le second cas, le corps de l'étranger banni trouve dans le sida une condamnation sans appel. La condition d'immigré et la condition de malade convergent dans une même logique d'exclusion. L'ordre public s'impose. La vie biologique disparait avec la vie sociale. Dans le troisième cas, le corps de l'étranger est mis à l'épreuve du sida. La condition d'immigré et la condition de malade deviennent les objets de la reconstruction du sujet. Le vivre avec apparait comme un principe de résistance à cette double contrainte. La vie sociale se réorganise autour de la vie biologique.

Que l'on ne se méprenne cependant pas sur cette représentation idéale typique: les trois figures, si elles procèdent de logiques politiques distinctes et donnent lieu à des expériences différentes, ne s'excluent pas. Peter Igun, lorsqu'il est vu la première fois, se vit lui-même au ban de l'humanité, sans territoire ni statut, n'ayant plus sa place en France de par la loi, ni au Nigeria à cause de la maladie. Karim El Mahdi, jusqu'au dernier entretien, manifeste une forme de résistance à la double violence de l'interdiction de séjour et de la menace de mort. Sophie Mugangu, à la fin de son histoire, a aussi trouvé la reconnaissance légale que lui confere l'autorisation provisoire de séjour. Le rapport entre les deux conditions n'est pas donc posé une fois pour toutes. C'est à chaque moment dans l'interaction entre l'État et les étrangers qu'il se constitue. Les changements législatifs, mais aussi les mobilisations sociales ne cessent de le déplacer.

À cet égard, on peut lire comme exemplaire des glissements entre ces figures l'histoire dont l'association Migrants contre le sida a fait l'emblème de son combat et dont les épisodes de la vie émaillent les messages pluri-hebdomadaires de leur site Internet : «Depuis trois ans, Ali B. est en lutte pour l'égalité des droits des malades du sida", peut-on lire dans un message envoyé le 20 mars 2000 et intitulé : «L'État veut la mort des malades». «Le 7 février 1997, Ali B., gravement malade, suivant une trithérapie, était expulsé vers la Tunisie car frappé par la double peine. Une forte mobilisation - en plein vote des lois Debré - avait 
abouti à son retour en France. Mais il s'est retrouvé comme avant l'expulsion, privé de tous droits sociaux et interdit de circuler car soumis à une assignation à résidence. Depuis il n'a jamais cessé de lutter pour sa dignité et celle de tous les autres qui affrontent, au quotidien, le sida et les pratiques qui tuent les malades étrangers. Cela aucun état raciste ne peut le supporter de la part d'un malade immigré. Aussi le soutien dont il a besoin aujourd'hui est celui de tous ceux qui seront capables de voir en lui un camarade et non un cas humanitaire... ". Au-delà des effets rhétoriques de cette dénonciation, on voit comment le corps banni devient, sous la pression des associations de lutte contre le sida, un corps légitime que la maladie force à reconnaître, avant de se muer en corps retrouvé dans le combat politique et la solidarité associative, les trois figures se superposant du reste plus qu'elles ne se substituent l'une à l'autre.

La tension entre ces figures s'inscrit elle-même dans un mouvement historique par lequel les politiques redéfinissent la condition de l'immigré (Fassin \& Morice 2001). L'immigration, en tant qu'elle est considérée avant tout, du point de vue des sociétés d'accueil contemporaines, comme un apport de maind'œuvre, a perdu depuis le début des années 70 beaucoup de son importance économique et, par conséquent, de sa légitimité sociale (Marie 1997). Les transformations du monde du travail et du marché de l'emploi, avec l'augmentation du chômage en particulier pour les métiers à faible qualification, en ont limité les bénéfices estimés à certains secteurs d'activité qui, du reste, ne se sont pas seulement accommodés de la précarisation juridique des immigrés, mais ont favorisé le développement du travail au noir, notamment dans le cadre de la sous-traitance. Double dévalorisation de la force de travail étrangère, donc, puisque, d'un côté, elle est présentée comme dénuée d'utilité sociale et même dangereuse pour la situation de l'emploi, et de l'autre, elle est essentiellement maintenue dans ses formes juridiquement les plus dégradées, au risque là encore de la faire apparaître comme une menace pour le monde du travail.

C'est dans ce contexte, où les immigrés sont devenus en quelque sorte physiquement indésirables - et l'on doit ici se souvenir de la récurrence de l'invocation des caractéristiques physiques dans les discours politiques sur l'immigration, bien au-delà de la seule question de leur contribution comme force de travail - qu'apparaît, au cours des années 70, une sorte de limite corporelle inédite à leur rejet : c'est l'autorisation de séjour pour soins, autrement dit la reconnaissance du droit à se maintenir sur le territoire français pour autant que l'on puisse faire la démonstration d'une atteinte grave du corps auquel le retour dans le pays ferait courir un risque vital. Dans le même temps, la citoyenneté se trouve restreinte à la seule dimension sociale, à l'exclusion donc de toute dimension politique. Leur est reconnu un ensemble de droits, qu'il s'agisse du revenu minimum d'insertion ou de l'accès aux soins, manifestant une préoccupation d'égalité devant les bénéfices attendus de l'État social, cependant que la participation à la vie de la cité se trouve systématiquement soustraite au débat public - que l'on songe à la question du vote des étrangers aux élections locales, constamment évoquée dans les programmes électoraux et jamais mise en chan- 
tier ensuite. Cette forclusion du politique trouve une traduction particulière dans l'instauration d'une procédure exceptionnelle de double pénalisation, sous la forme d'un emprisonnement associé à un bannissement, dont les étrangers ont l'exclusivité. Au fond, la simple existence de cette disposition légale signifie le peu de cas politique que l'on fait de l'immigré.

Si l'on reprend la distinction, précédemment discutée, des deux termes grecs signifiant la vie, on peut désormais esquisser les éléments de ce que l'on pourrait appeler les biopolitiques de l'immigration (Fassin 2001), en référence explicite à l'analyse de Michel Foucault quoique dans une perspective quelque peu différente.

Au premier sens, zôé, qui correspond à la forme élémentaire de la vie, "processus vital " pour Hannah Arendt (1983: 145), «simple fait de vivre" pour Giorgio Agamben (1997: 9), la condition de l'immigré se voit déplacée d'une reconnaissance de la vie comme force de travail vers une reconnaissance de la vie comme phénomène biologique. Réduction ultime de l'humain à son expression physique : inutile et indésirable en tant que travailleur, l'étranger conserve une légitimité en tant que malade. À ce titre, il pourra rester sur le territoire national et y bénéficier de soins le plus souvent gratuits. Évolution significative par rapport à un temps où, comme l'analyse marxiste l'avait montré, l'attention au corps des dominés avait pour objet de maintenir leur productivité et donc d'accroître leur exploitation. C'est ainsi que Michel Foucault (1976) justifiait l'émergence, à la fin du XVIII siècle, d'un «bio-pouvoir » au service du capitalisme. Dans le contexte contemporain, au contraire, le rendement de la bio-politique en matière d'immigration est marginal. La logique n'est pas économique, elle est essentiellement humanitaire.

Au second sens, bios, qui correspond à l'existence en société, «spécifiquement humaine " pour Hannah Arendt (1983 : 143), « façon de vivre propre à un individu ou à un groupe " pour Giorgio Agamben (1997 : 9), la condition de l'immigré se trouve réduite à une citoyenneté sociale sans contrepartie civique. Dans sa manifestation la plus répressive, la négation de l'étranger comme sujet politique donne lieu au régime d'exception de l'interdiction de territoire conduisant à une apatridie de fait. Loin d'être accessoire, si l'on tient compte du nombre de personnes concernées et de la hiérarchie implicite des valeurs qui la rend presque inéluctable, y compris en cas de maladie grave, cette disposition légale vient rappeler le statut précaire de l'étranger, sa légitimité toujours révisable. Pour autant, l'existence sociale de l'immigré ne se donne pas à lire tout entière dans cette forme d'assujettissement à la loi du pays. Elle s'exprime aussi dans les modes de subjectivation que les patients développent, notamment à travers les ressources que, paradoxalement, leur corps éprouvé leur fournit. Ces espaces de liberté, qui manquaient à sa conception initiale du pouvoir, Michel Foucault (1994) avait essayé de leur donner droit de cité dans son analyse ultérieure en termes de "gouvernementalité ». De ce point de vue, l'étranger, même en situation irrégulière et même malade du sida, n'est pas seulement une victime : il est aussi, quoique sous fortes contraintes, un acteur social et un sujet politique. 
Dans sa remarquable enquête sur la tuberculose parmi les populations noires d'Afrique du Sud, White Plague, Black Labour, l'historien Randall Packard 160 (1989) montrait comment la conjonction d'une réalité épidémique et d'une situation de domination rendait nécessaire une économie politique de la santé. Rejetant les interprétations qui mettaient en avant les dimensions génétique ou culturelle de cette affection, il en révélait les déterminations sociales, économiques et politiques, dans le contexte de la colonisation puis de l'apartheid. Le sida, dans les sociétés contemporaines, appelle le même réexamen, en particulier lorsqu'on s'efforce d'appréhender le développement de l'infection et l'expérience de la maladie parmi les populations étrangères ou les minorités ethniques. Mais s'il faut assurément considérer les conditions sociales, économiques et politiques qui sous-tendent l'un et l'autre phénomène (épidémiologique et sociologique), on peut aussi s'interroger sur la manière dont la condition d'immigré interfere avec la condition de malade et sur la signification de la relation entre les deux. Au-delà de ce qu'elle nous apprend de la situation des immigrés malades, la condition sociale ainsi définie devient alors un analyseur des fondements moraux de notre monde.

MOTS CLÉS/KEYWORDS: immigration/immigration - sida/aids - biopolitique/biopolitics anthropologie médicale/medical anthropology.

BIBLIOGRAPHIE

Agamben, Giorgio

Becker, Howard S.

1997 Homo sacer. Le pouvoir souverain et la vie nue. Paris, Le Seuil (1 ${ }^{\mathrm{re}}$ éd. italienne 1995).

Alonzo, Angelo A. \& Nancy R. Reynolds 1995 "Stigma, HIV and AIDS : An Exploration and Elaboration of a Stigma Trajectory ", Social Science and Medicine 41 (3) : 303-315.

Anderson, Robert \& Michael Bury 1988 Living with Chronic Illness. The Experience of Patients and their Families. Londres, Unwyn Hyman.

Arendt, Hannah

1982 Les Origines du totalitarisme.

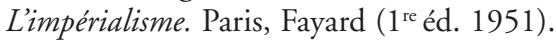

1983 Condition de l'homme moderne. Paris, Calmann-Lévy (1 ${ }^{\text {re }}$ éd. 1958).

1985 Outsiders. Études de sociologie de la déviance. Paris, Métailié (1 ${ }^{\text {re }}$ éd. 1963).

Bergeron, Henri

1999 L'État et la toxicomanie. Histoire d'une singularité. Paris, PUF ("Sociologies»).

Castel, Robert

1995 Les Métamorphoses de la question sociale. Une chronique du salariat. Paris, Fayard.

Certeau, Michel de

1980 L'Invention du quotidien. Arts de faire. Paris, UGE.

Clatts, Michael C. \& Kevin M. Mutchler 1989 «AIDS and the Dangerous Other : Metaphors of Sex and Deviance in the 
Représentation of Disease ", Medical Anthropology 10 : 105-114.

\section{Delaunay, Karine}

1999 « Des groupes à risque à la vulnérabilité des populations africaines. Discours sur une pandémie ", Autrepart $12: 37-52$.

\section{Farmer, Paul}

1997 «On Suffering and Structural Violence. A View from Below", in Arthur Kleinman, Veena Das \& Margaret Lock, eds, Social Suffering. Berkeley, University of California Press : 261-283. 1999 "AIDS and Social Scientists. Critical Reflections " in Charles Becker, Jean-Pierre Dozon, C. Obbo \& M. Touré, eds, Vivre et penser le sida en Afrique. Paris, Karthala : 33-39.

\section{Farmer, Paul \& Arthur Kleinman}

1989 "AIDS as Human Suffering", Daedalus 118 (2) : 135-160.

\section{Fassin, Didier}

1996a "Idéologie, pouvoir et maladie. Éléments d'anthropologie politique du sida en Afrique ", in Michèle Cros, ed., Les Maux de l'Autre. Paris, L'Harmattan : 65-93.

$1996 b$ "Secret, civisme et citoyenneté. La dénonciation des étrangers en situation irrégulière ", Agora. Éthique, médecine, société 37 : 33-39.

1999a «L'indicible et l'impensé. La "question immigrée" dans les politiques du sida ", Sciences sociales et Santé 17 (4) : 5-36. $1999 \mathrm{~b}$ "Santé et immigration. Les vérités politiques du corps", Cahiers de l'URMIS 5 : 69-76.

2001 «The Biopolitics of Otherness. Undocumented Foreigners and Racial Discrimination in the French Public Debate ", Anthropology Today 17 (1) : 3-7.

\section{Fassin, Didier \& Alain Morice}

2001 «Les épreuves de l'irrégularité. Les sans-papiers, entre déni d'existence et reconquête d'un statut ", in Dominique Schnapper ed., Exclusions au cour de la cité. Paris, Anthropos : 260-309.

\section{Foucault, Michel}

1976 Histoire de la sexualité. La volonté de savoir. Paris, Gallimard.

1994 Dits et écrits. Vol. 4, Paris, Gallimard.

\section{Hahn, Alaïs, Willy H. Eirmbter \&}

Rüdiger Jacob

1994 «Le sida : savoir ordinaire et insécurité ", Actes de la Recherche en Sciences sociales 104 : 81-89.

\section{Haour-Knipe, Mary \& Richard Rector}

1996 Crossing Borders. Migration, Ethnicity and AIDS. Londres, Taylor \& Francis.

\section{Kleinman, Arthur}

1995 Writing at the Margin. Discourse Between Anthropology and Medicine.

Berkeley, University of California Press.

\section{Lot, Florence}

2000 «Les données sur le sida dans la population étrangère en France", Hommes et migrations, $\mathrm{n}^{\circ}$ spécial 1225 : Santé: le traitement de la différence: 83-87.

\section{Marie, Claude-Valentin}

1997 «À quoi sert l'emploi des étrangers?" ", in Didier Fassin, Alain Morice \& Catherine Quiminal, eds, Les Lois de l'inhospitalité. Les politiques de l'immigration à l'épreuve des sans-papiers. Paris, La Découverte : 145-175.

\section{Packard, Randall M.}

1989 White Plague, Black Labor:

Tuberculosis and the Political Economy of Health and Disease in South Africa.

Berkeley, University of California Press.

\section{Palidda, Salvatore}

1999 "La criminalisation des migrants", Actes de la Recherche en Sciences sociales $129: 39-49$.

\section{Pollak, Michael}

1990 L'Expérience concentrationnaire. Essai sur le maintien de l'identité sociale. Paris, Métailié. 
Quiminal, Catherine

1991 Gens d'ici, gens d'ailleurs. Migrations

162 soninké et transformations villageoises. Paris, Christian Bourgois.

\section{Rabinow, Paul}

1996 Essays on the Anthropology of Reason. Princeton, Princeton University Press.

Sayad, Abdelmalek

1999a La Double absence. Des illusions de l'émigré aux souffrances de l'immigré. Paris, Le Seuil.
1999 b "Immigration et pensée d'État ", Actes de la Recherche en Sciences sociales 129: 5-14.

\section{Spire, Alexis}

1999 «De l'étranger à l'immigré. La magie sociale d'une catégorie statistique ", Actes de la Recherche en Sciences sociales 129: 50-56.

Trachet, Françoise \& Mamadou Alioune Diallo 1994 «Les représentations de l'infection à VIH et de la maladie du sida dans une population migrante d'Afrique de l'Ouest", Migrations et Santé 80 : 75-89. 